\title{
micromachines
}

ISSN 2072-666X

www.mdpi.com/journal/micromachines

Article

\section{Effect of Laser-Induced Heating on Raman Measurement within a Silicon Microfluidic Channel}

\section{Ying Lin ${ }^{1}$, Xinhai Yu ${ }^{2, *}$, Zhenyu Wang ${ }^{3}$, Shan-Tung Tu ${ }^{2}$ and Zhengdong Wang ${ }^{2}$}

1 School of Mechanical Engineering, Shanghai Institute of Technology, Shanghai 201418, China; E-Mail: rememberit@163.com

2 Key Laboratory of Pressurized Systems and Safety, Ministry of Education, School of Mechanical and Power Engineering, East China University of Science and Technology, Shanghai 200237, China; E-Mails: sttu@ecust.edu.cn (S.-T.T.); zdwang@ecust.edu.cn (Z.W.)

3 School of Software and Microelectronics at Wuxi, Peking University, Wuxi 214125, China; E-Mail: zw@ss.pku.edu.cn

* Author to whom correspondence should be addressed; E-Mail: yxhh@ecust.edu.cn; Tel.: +86-21-6425-3708; Fax: +86-21-6425-3708.

Academic Editor: Jeong-Bong Lee

Received: 24 April 2015 / Accepted: 19 June 2015 / Published: 29 June 2015

\begin{abstract}
When Raman microscopy is adopted to detect the chemical and biological processes in the silicon microfluidic channel, the laser-induced heating effect will cause a temperature rise in the sample liquid. This undesired temperature rise will mislead the Raman measurement during the temperature-influencing processes. In this paper, computational fluid dynamics simulations were conducted to evaluate the maximum local temperature-rise (MLT). Through the orthogonal analysis, the sensitivity of potential influencing parameters to the MLT was determined. In addition, it was found from transient simulations that it is reasonable to assume the actual measurement to be steady-state. Simulation results were qualitatively validated by experimental data from the Raman measurement of diffusion, a temperature-dependent process. A correlation was proposed for the first time to estimate the MLT. Simple in form and convenient for calculation, this correlation can be efficiently applied to Raman measurement in a silicon microfluidic channel.
\end{abstract}

Keywords: laser-induced heating; Raman measurement; microchannel; computational fluid dynamics 


\section{Introduction}

The last decade has witnessed an explosive growth in applications of microfluidic channels to chemical and biological fields [1-5]. This has been driven by the trends of shrinking conventional benches to a small size to realize major advantages of efficiency, performance, integration, speed and cost. Microfluidics was established during the advance of microelectronics. Silicon was the representative material of the microelectronics, and then was directly utilized for microfluidics. It is indispensable for the specialized systems that require mechanical, chemical and thermal stability.

In the application of microfluidic systems, the Raman microscope has proved to be useful to perform in situ monitoring of chemical and biochemical processes [6-10]. Especially, novel surface-enhanced Raman scattering has greatly extended the role of the standard Raman microscope [11-14]. Commercial Raman microscopes are usually configured with metallurgical objectives that are designed to work in air. In this case, the laser beam focused on the liquid will suffer the strong refraction at the air-chip interface $[15,16]$, leading to the focus distortion. On one hand, the distorted focus will contribute to unfavorable out-of-focus signals, which lowers the accuracy of measurements in the microfluidic channel. Microchannels with high aspect ratio (width/height) and small height were routinely used to deal with this problem, because in so doing, Raman signals would originate from a region with uniform concentration [9,17]. On the other hand, when focusing very close to the bottom of the microchannel, the distorted focus will reach the channel bottom. The heating effect is therefore believed to occur due to the absorption of the substrate material to the incident laser energy. For an opaque substrate material such as silicon, a considerable amount of the incident visible light power is absorbed, resulting in an appreciable local liquid temperature rise. This phenomenon has been demonstrated in our previous work [18].

The laser-induced heating is noticeable in Raman measurement, because the local increased temperature will give rise to Raman temperature shift $[19,20]$. In the application of Raman stress measurement, the stress was analyzed through Raman shift. However, the unexpectedly small temperature shift produced by local heating concealed the stress shift and thus failed the stress measurement $[21,22]$. These findings necessitate detailed investigations on the laser induced heating effect, as well as effective approaches to estimate and eliminate this effect. To study the effect, numerical simulation, a simple and direct method, is commonly used in various applications, i.e., laser machining [23] and tip-enhanced optical detection [24]. In addition, laser-induced heating has also been utilized to assist biological process [25], which benefits from a small heating region controlled by laser spot. The precise temperature detection and calibration rely on microsensor [26,27] or optical imaging technique [28]. Although many studies have focused on the laser-induced heating effect, the effect occurring in the solid and fluid flow, a more complicated problem, was found in our previous work. However, a large number of parameters are involved in the flow and measurement system. The contribution of each parameter to the heating effect needs to be further investigated. It is expected to estimate this effect before experiments, and some approaches can be made to decrease the temperature rise if the heating effect exists.

In order to qualify the laser-induced heating effect within a silicon microchannel, computational fluid dynamic (CFD) simulations were conducted in this paper. Several parameters involved in the flow and measurement system were selected. By using the orthogonal array, the sensitivity of these 
parameters to the temperature rise was studied. The most sensitive parameters were separately investigated to present their relations to the temperature rise. To substantiate the CFD results, diffusion, a temperature-influenced process, was experimentally investigated. A correlation was proposed to estimate the heating effect based on the dimensional and regression analysis. This correlation provides a convenient approach to quantify the consequent temperature rise.

\section{Numerical Simulations}

\subsection{Preliminaries Prior to Modeling}

Prior to modeling of the heating effect, the condition under which the heating effect cannot be neglected has to be specifically determined. The laser-induced heating effect occurs when the Raman laser beam is focused on the liquid very close to the channel bottom. To be more specific, the heating effect is believed to exist when the focus depth of the Raman laser is comparable with the distance between the focus center and the channel bottom. The focus depth can be obtained from the full-width-at-half-maximum (FWHM) of the intensity profile by depth-scanning [15,29,30]. It can also be predicted by several theoretical or empirical models [16,31,32].

As stated previously, the local heating results from the silicon's absorption to the laser that reaches the silicon surface. Therefore, the heating effect can be simulated by exerting a power density in the form of heat flux on the silicon surface. This heat flux is related to the power at the silicon surface (termed surface power), the absorption coefficient and the laser illuminated area of the surface. The actual surface power can be easily measured by a laser power meter. The absorption coefficient of silicon to the $514.5 \mathrm{~nm}$ incident light $\alpha$ is $65 \%$. This is indirectly obtained by measuring the reflection coefficient through UV-VIS-NIR spectrophotometer (Varian Cary 500, Varian Medical Systems, Inc, Palo Alto, CA, USA). Furthermore, the laser illuminated area was conservatively estimated by the laser spot diameter directly measured on the silicon surface. The pertinent equations for calculating the exerted heat flux are presented in the following sections. The transparent fluids were considered, thus their absorption to the incident light was neglected.

\subsection{Physical Model, Computational Equations and Boundary Conditions}

To model the heating phenomenon, the conjugate heat transfer and fluid flow were numerically solved by the commercial software ANSYS CFX 11.0 (ANSYS, Inc, Canonsburg, PA, USA). A three-dimensional physical model for the silicon/glass microfluidic chip was established, as depicted in Figure 1. The model was described with the following assumptions:

(1) Steady-state (its reasonableness is discussed in Section 4.4), laminar and incompressible flow;

(2) Negligible radiation heat transfer;

(3) Negligible buoyancy;

(4) Constant fluid and solid (silicon) properties;

(5) Negligible viscous heating;

(6) Pyrex glass layer was not explicitly modeled due to its low thermal conductivity;

(7) Only half of the geometry was represented due to the symmetry of the domain;

(8) Gaussian laser beam profile. 


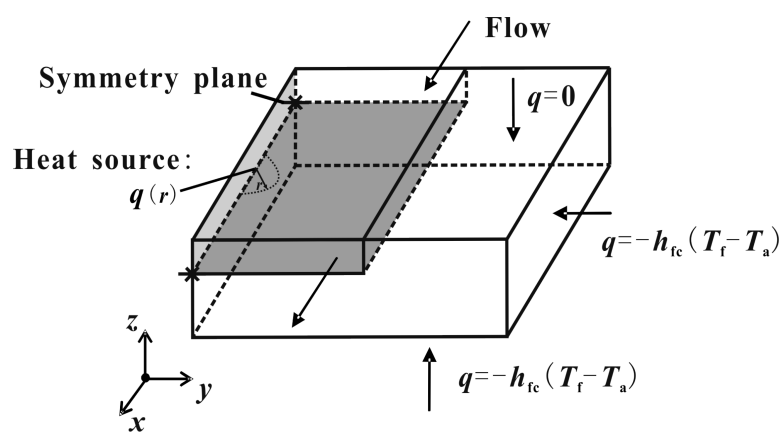

Figure 1. Physical model of the laser-induced heating effect. The heat source is a heat flux with Gaussian profile as shown in Equation (8). The Gaussian profile is determined by $P, \alpha$ and $d$. In our case, $\alpha=65 \%$ and $P$ and $d$ range from $0.5-10 \mathrm{~mW}$ and $1-5 \mu \mathrm{m}$, respectively. The mesh within a $10 \mu \mathrm{m}$ diameter spherical space around the heat source center was refined up to $0.02 \mu \mathrm{m}$.

Under the stated assumptions, the conservation equations are:

Mass (continuity) equation:

$$
\nabla \cdot \vec{u}=0
$$

Momentum equation:

$$
\rho_{\mathrm{f}}(\vec{u} \cdot \nabla \vec{u})=-\nabla p+\mu_{\mathrm{f}} \nabla^{2} \vec{u}
$$

Energy equation for the fluid:

$$
\rho_{\mathrm{f}} c_{p \mathrm{f}}(\vec{u} \cdot \nabla T)=\lambda_{\mathrm{f}} \nabla^{2} T
$$

Energy equation for the solid:

$$
\lambda_{\mathrm{s}} \nabla^{2} T=0
$$

Based on the assumptions of constant fluid properties and negligible buoyancy, the mass and momentum equations were not coupled with the energy equations. Therefore, the temperature field was calculated by solving the energy equation after the converged solution for the flow field was obtained. Such an approach allowed to significantly speed up the computational convergence. The momentum and mass equations were solved for the following hydraulic boundary conditions:

- Uniform velocity profile at the inlet (the difference between the uniform and parabolic assumption has been proven to be negligible in our case);

- Constant pressure of 1 bar at the outlet;

- Non-Slip boundary conditions at all walls.

The thermal boundary conditions for the fluid were set as follows:

- Constant temperature $(T=296.15 \mathrm{~K})$ at the inlet and $\mathrm{d} T / \mathrm{d} x=0$ at the outlet;

- Adiabatic condition at the fluid-Pyrex boundary (top of the chip);

- The continuity of the temperature and heat flux is automatically used in the CFX code as conjugate boundary conditions to couple the energy equations for the fluid and solid phases. It is defined as Equation (5) at the fluid-solid interface. 


$$
-\lambda_{\mathrm{s}}\left(\left.\frac{\partial T_{\mathrm{s}}(x, y, \mathrm{z})}{\partial n}\right|_{\Gamma}\right)=-\lambda_{\mathrm{f}}\left(\left.\frac{\partial T_{\mathrm{f}}(x, y, \mathrm{z})}{\partial n}\right|_{\Gamma}\right)
$$

The thermal boundary conditions for the solid were set as follows:

- Heat source: a heat flux centered on the solid side of the fluid-solid interface (marked in Figure 1). The heat flux was specified as the absorbed energy with a function of $r$. The function was conducted from the laser beam intensity form with Gaussian profile:

$$
I(r)=I_{0} \exp \left(-\frac{r^{2}}{\omega_{0}^{2}}\right)
$$

where $\omega_{0}$ is defined by $I\left(\omega_{0}\right)=I_{0} / e$, and the diameter of illuminated area defined in our study is $d=2 \omega_{0}$. Then, the total laser power at the surface is:

$$
P=2 \pi \int_{0}^{\infty} r I(r) \mathrm{d} r=\pi \omega_{0}^{2} I_{0}
$$

- The energy absorbed in the solid is:

$$
q(r)=I_{0} \alpha \exp \left(-\frac{r^{2}}{\omega_{0}^{2}}\right)=\frac{P \alpha}{\pi \omega_{0}^{2}} \exp \left(-\frac{r^{2}}{\omega_{0}^{2}}\right)
$$

The heat source was exerted on the solid side of the horizontal flui-dsolid interface in the form of

$$
-\lambda_{\mathrm{s}} \frac{\partial T}{\partial z}=q
$$

- Adiabatic condition at the silicon-Pyrex boundary;

- Free convective heat transfer with surrounding air at the lateral walls and underside of the solid. Both the ambient and initial solid temperature was $296.15 \mathrm{~K}$. The free convective heat transfer coefficient $h_{\mathrm{fc}}$ was estimated at $10 \mathrm{~W} \cdot \mathrm{m}^{-2} \cdot \mathrm{K}^{-1}$. This $h_{\mathrm{fc}}$ value is close enough due to the relatively minor effect of the heat loss [33].

The three-dimensional numerical simulations were developed based on the finite-volume scheme. The mesh was composed of tetrahedral elements. The element sizes were $8 \mu \mathrm{m}$ in the fluid domain and $25 \mu \mathrm{m}$ in the solid domain. The elements within a $10 \mu \mathrm{m}$ diameter spherical space around the heat source were refined up to $0.02 \mu \mathrm{m}$ to capture a drastic temperature change in this space. No significant change in the simulation results was observed even if the element number was doubled.

\subsection{Methodology of Orthogonal Array (OA) Analysis}

The laser-induced heating directly engenders the local temperature rise. The maximum local temperature-rise (MLT), defined as the highest value of temperature rise in the fluid domain, is taken as the measure of the laser-induced heating effect in this paper. Several parameters were considered potential factors that will influence this heating effect. In order to determine the various extents to which these parameters affected the MLT, the sensitivity analysis was carried out by means of the orthogonal array (OA). First, the Raman laser parameters, i.e., the surface power $P$ and the diameter of illuminated area $d$, were selected as OA factors. Second, the forced convective heat transfer was 
considered since it might have an essential effect on the heating. Thus, the fluid mean velocity, fluid physical properties and microchannel sizes were selected. Third, the thermal conduction heat transfer in the solid may be comparable with the forced convective heat transfer, due to the large solid domain sizes and the high thermal conductivity of the solid substrate. Hence, the geometric parameters of the chip were included in the OA to investigate the contribution of the thermal conduction. In this way, 11 design parameters (factors) related to the laser-induced heating effect were determined. Then, a table of 50 designs was generated through the orthogonal array (OA) L50 (see Table 1). To match with this table, five levels were selected, and their values were given, as listed in Table 2. Liquid physical properties were obtained from the database of some common liquids [34]. The conjugate heat transfer and fluid flow through 50 different designed conditions were then simulated by CFD to yield the MLT.

Through the OA, the most influencing factors on the heating effect can be identified. Those factors were separately investigated in detail to quantitatively reveal their relations to the heating effect in this paper.

Table 1. Orthogonal Array L50 (design matrix).

\begin{tabular}{|c|c|c|c|c|c|c|c|c|c|c|c|c|c|c|c|c|c|c|c|c|c|c|c|}
\hline \multirow{2}{*}{ Simulations } & \multicolumn{11}{|c|}{ Factors } & \multirow{2}{*}{ Simulations } & \multicolumn{11}{|c|}{ Factors } \\
\hline & A & $\mathrm{B}$ & $\mathrm{C}$ & $\mathrm{D}$ & $\mathrm{E}$ & $\mathrm{F}$ & G & $\mathrm{H}$ & I & $\mathrm{J}$ & $\mathrm{K}$ & & A & $\mathrm{B}$ & $\mathrm{C}$ & $\mathrm{D}$ & $\mathrm{E}$ & $\mathrm{F}$ & $\mathrm{G}$ & $\mathrm{H}$ & I & $\mathrm{J}$ & $\mathrm{K}$ \\
\hline No. 1 & 1 & 1 & 1 & 1 & 1 & 1 & 1 & 1 & 1 & 1 & 1 & No. 26 & 1 & 1 & 1 & 4 & 5 & 4 & 3 & 2 & 5 & 2 & 3 \\
\hline No. 2 & 1 & 2 & 2 & 2 & 2 & 2 & 2 & 2 & 2 & 2 & 2 & No. 27 & 1 & 2 & 2 & 5 & 1 & 5 & 4 & 3 & 1 & 3 & 4 \\
\hline No. 3 & 1 & 3 & 3 & 3 & 3 & 3 & 3 & 3 & 3 & 3 & 3 & No. 28 & 1 & 3 & 3 & 1 & 2 & 1 & 5 & 4 & 2 & 4 & 5 \\
\hline No. 4 & 1 & 4 & 4 & 4 & 4 & 4 & 4 & 4 & 4 & 4 & 4 & No. 29 & 1 & 4 & 4 & 2 & 3 & 2 & 1 & 5 & 3 & 5 & 1 \\
\hline No. 5 & 1 & 5 & 5 & 5 & 5 & 5 & 5 & 5 & 5 & 5 & 5 & No. 30 & 1 & 5 & 5 & 3 & 4 & 3 & 2 & 1 & 4 & 1 & 2 \\
\hline No. 6 & 2 & 1 & 2 & 3 & 4 & 5 & 1 & 2 & 3 & 4 & 5 & No. 31 & 2 & 1 & 2 & 1 & 3 & 3 & 2 & 4 & 5 & 5 & 4 \\
\hline No. 7 & 2 & 2 & 3 & 4 & 5 & 1 & 2 & 3 & 4 & 5 & 1 & No. 32 & 2 & 2 & 3 & 2 & 4 & 4 & 3 & 5 & 1 & 1 & 5 \\
\hline No. 8 & 2 & 3 & 4 & 5 & 1 & 2 & 3 & 4 & 5 & 1 & 2 & No. 33 & 2 & 3 & 4 & 3 & 5 & 5 & 4 & 1 & 2 & 2 & 1 \\
\hline No. 9 & 2 & 4 & 5 & 1 & 2 & 3 & 4 & 5 & 1 & 2 & 3 & No. 34 & 2 & 4 & 5 & 4 & 1 & 1 & 5 & 2 & 3 & 3 & 2 \\
\hline No. 10 & 2 & 5 & 1 & 2 & 3 & 4 & 5 & 1 & 2 & 3 & 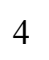 & No. 35 & 2 & 5 & 1 & 5 & 2 & 2 & 1 & 3 & 4 & 4 & 3 \\
\hline No. 11 & 3 & 1 & 3 & 5 & 2 & 4 & 4 & 1 & 3 & 5 & 2 & No. 36 & 3 & 1 & 3 & 3 & 1 & 2 & 5 & 5 & 4 & 2 & 4 \\
\hline No. 12 & 3 & 2 & 4 & 1 & 3 & 5 & 5 & 2 & 4 & 1 & 3 & No. 37 & 3 & 2 & 4 & 4 & 2 & 3 & 1 & 1 & 5 & 3 & 5 \\
\hline No. 13 & 3 & 3 & 5 & 2 & 4 & 1 & 1 & 3 & 5 & 2 & 4 & No. 38 & 3 & 3 & 5 & 5 & 3 & 4 & 2 & 2 & 1 & 4 & 1 \\
\hline No. 14 & 3 & 4 & 1 & 3 & 5 & 2 & 2 & 4 & 1 & 3 & 5 & No. 39 & 3 & 4 & 1 & 1 & 4 & 5 & 3 & 3 & 2 & 5 & 2 \\
\hline No. 15 & 3 & 5 & 2 & 4 & 1 & 3 & 3 & 5 & 2 & 4 & 1 & No. 40 & 3 & 5 & 2 & 2 & 5 & 1 & 4 & 4 & 3 & 1 & 3 \\
\hline No. 16 & 4 & 1 & 4 & 2 & 5 & 3 & 5 & 3 & 1 & 4 & 2 & No. 41 & 4 & 1 & 4 & 5 & 4 & 1 & 2 & 5 & 2 & 3 & 3 \\
\hline No. 17 & 4 & 2 & 5 & 3 & 1 & 4 & 1 & 4 & 2 & 5 & $J$ & No. 42 & 4 & 2 & 5 & 1 & 5 & 2 & 3 & 1 & 3 & 4 & 4 \\
\hline No. 18 & 4 & 3 & 1 & 4 & 2 & 5 & 2 & 5 & 3 & 1 & 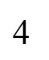 & No. 43 & 4 & 3 & 1 & 2 & 1 & 3 & 4 & 2 & 4 & 5 & 5 \\
\hline No. 19 & 4 & 4 & 2 & 5 & 3 & 1 & 3 & 1 & 4 & 2 & $J$ & No. 44 & 4 & 4 & 2 & 3 & 2 & 4 & 5 & 3 & 5 & 1 & 1 \\
\hline No. 20 & 4 & 5 & 3 & 1 & 4 & 2 & 4 & 2 & 5 & 3 & . & No. 45 & 4 & 5 & 3 & 4 & 3 & 5 & 1 & 4 & 1 & 2 & 2 \\
\hline No. 21 & 5 & 1 & 5 & 4 & 3 & 2 & 4 & 3 & 2 & 1 & 3 & No. 46 & 5 & 1 & 5 & 2 & 2 & 5 & 3 & 4 & 4 & 3 & 1 \\
\hline No. 22 & 5 & 2 & 1 & 5 & 4 & 3 & 5 & 4 & 3 & 2 & 1 & No. 47 & 5 & 2 & 1 & 3 & 3 & 1 & 4 & 5 & 5 & 4 & 2 \\
\hline No. 23 & 5 & 3 & 2 & 1 & 5 & 4 & 1 & 5 & 4 & 3 & 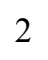 & No. 48 & 5 & 3 & 2 & 4 & 4 & 2 & 5 & 1 & 1 & 5 & 3 \\
\hline No. 24 & 5 & 4 & 3 & 2 & 1 & 5 & 2 & 1 & 5 & 4 & 3 & No. 49 & 5 & 4 & 3 & 5 & 5 & 3 & 1 & 2 & 2 & 1 & 4 \\
\hline No. 25 & 5 & 5 & 4 & 3 & 2 & 1 & 3 & 2 & 1 & 5 & 4 & No. 50 & 5 & 5 & 4 & 1 & 1 & 4 & 2 & 3 & 3 & 2 & 5 \\
\hline
\end{tabular}

Notes: A: Surface power, i.e., the power at the sample surface, $P(\mathrm{~mW})$. B: Diameter of illuminated area, $d(\mu \mathrm{m})$. C: Mean velocity, $U\left(\mathrm{~m} \cdot \mathrm{s}^{-1}\right)$. D: Density of the fluid, $\rho_{\mathrm{f}}\left(\mathrm{kg} \cdot \mathrm{m}^{-3}\right)$. E: Viscosity of the fluid, $\mu_{\mathrm{f}}(\mathrm{mPa} \cdot \mathrm{s})$. F: Specific heat of the fluid, $c_{p \mathrm{f}}\left(\mathrm{kJ} \cdot \mathrm{kg}^{-1} \cdot \mathrm{K}^{-1}\right)$. G: Thermal conductivity of the fluid, $\lambda_{\mathrm{f}}\left(\mathrm{W} \cdot \mathrm{m}^{-1} \cdot \mathrm{K}^{-1}\right)$. H: Height of the microchannel, $h(\mu \mathrm{m})$. I: Width of the microchannel, $w(\mu \mathrm{m})$. J: Area of the microfluidic chip, $L \cdot W\left(\mathrm{~mm}^{2}\right)$. K: Length-to-width ratio of the microfluidic chip, $L / W$. 
Table 2. Factors and levels used in the orthogonal array (OA) L50 (design matrix).

\begin{tabular}{cccccccccccc}
\hline \multirow{2}{*}{ Level } & \multicolumn{10}{c}{ Factors } \\
\cline { 2 - 12 } & A & B & C & D & E & F & G & H & I & J & K \\
\hline 1 & 0.5 & 1 & 0.01 & 494 & 0.1 & 1.05 & 0.104 & 30 & 100 & 25 & 0.49 \\
2 & 1 & 2 & 0.025 & 867 & 0.55 & 1.72 & 0.151 & 60 & 300 & 64 & 0.64 \\
3 & 2.5 & 3 & 0.05 & 997 & 0.89 & 2.36 & 0.268 & 80 & 500 & 100 & 1 \\
4 & 5 & 4 & 0.075 & 1261 & 1.53 & 3.06 & 0.285 & 100 & 600 & 144 & 1.5625 \\
5 & 10 & 5 & 0.1 & 1584 & 1.92 & 4.19 & 0.6069 & 120 & 800 & 225 & 1.96 \\
\hline
\end{tabular}

Notes: A: Surface power, i.e., the power at the sample surface, $P(\mathrm{~mW})$. B: Diameter of illuminated area, $d(\mu \mathrm{m})$. C: Mean velocity, $U\left(\mathrm{~m} \cdot \mathrm{s}^{-1}\right)$. D: Density of the fluid, $\rho_{\mathrm{f}}\left(\mathrm{kg} \cdot \mathrm{m}^{-3}\right)$. E: Viscosity of the fluid, $\mu_{\mathrm{f}}(\mathrm{mPa} \cdot \mathrm{s})$. F: Specific heat of the fluid, $c_{p \mathrm{f}}\left(\mathrm{kJ} \cdot \mathrm{kg}^{-1} \cdot \mathrm{K}^{-1}\right)$. G: Thermal conductivity of the fluid, $\lambda_{\mathrm{f}}\left(\mathrm{W} \cdot \mathrm{m}^{-1} \cdot \mathrm{K}^{-1}\right)$. H: Height of the microchannel, $h(\mu \mathrm{m})$. I: Width of the microchannel, $w(\mu \mathrm{m})$. J: Area of the microfluidic chip, $L \cdot W\left(\mathrm{~mm}^{2}\right)$. K: Length-to-width ratio of the microfluidic chip, $L / W$.

\section{Experimental Section}

The heat flux induced by the laser is exerted on a small area of several square microns. The heat source will produce an MLT in the laser focus region, along with a sharp temperature gradient in the neighboring space. In such a small region, the accurate temperature measurement is difficult to achieve. Thereby, it is reasonable to select a temperature-influenced process to reflect the heating effect. To our knowledge, the diffusion process is temperature-dependent and is also convenient to perform in a straight microchannel $[1,17]$. Thus, the diffusion experiment was carried out to validate the simulation results.

A Y-shaped microchannel was fabricated on a silicon substrate by photolithography and deep reactive ion etching (DRIE). The rectangular cross section of the microchannel is $800 \mu \mathrm{m}$ wide and $30 \mu \mathrm{m}$ high. The substrate was then enclosed by the $500 \mu \mathrm{m}$ thick Pyrex glass wafer using anodic bonding.

Toluene and cyclohexane system was used in the diffusion measurement as a model. An initial toluene molar fraction difference $\Delta c$ in the order of 0.2 between two inlet liquids was used, as is typical. Under such a condition, the concentration dependence of the diffusion coefficient $(D)$ could be ignored so that the $D$ correlation process was simplified (for more details see our previous work [18]). Two solutions with the average concentrations of $c=0.7$ were separately injected into two inlets by one syringe pump (Cole-Parmer dual-syringe infusion pump, Vernon Hills, IL, USA). The experiments were in operation at $298 \mathrm{~K}$.

A confocal Raman microscope (Renishaw inVia microscope, New Mills, UK) was adopted to obtain the concentration data that were then used to correlate $D$ values. The entire system is schematically displayed in Figure 2. An argon ion laser (wavelength $=514.5 \mathrm{~nm}$ ) was scanned through an objective lens (Leica 50× ultra-long working distance). Using the full laser power of $25 \mathrm{~mW}$, the surface power $P$ was measured as $5 \mathrm{~mW}$ by the handheld power meter (Coherent, Santa Clara, CA, USA). The diameter of illuminated area was about $1 \mu \mathrm{m}$, obtained from the microscopic image by focusing the laser on the silicon surface. When the diffusive samples were flowing in the microchannel, the focus depth of $35 \mu \mathrm{m}$ was obtained from the FWHM of the intensity profile by depth-scanning. 


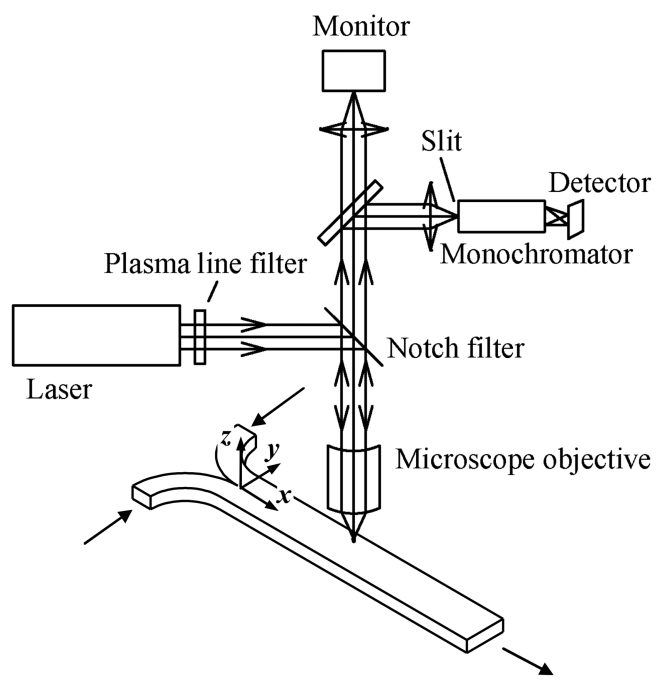

Figure 2. Schematic of the diffusion experiment system.

\section{Results and Discussion}

\subsection{General}

The heating effect was measured by the MLT, and it was therefore necessary to illustrate the location of the MLT as well as the temperature profile. Figure 3 presents the temperature profile at a certain condition. In Figure $3 \mathrm{a}$, the red region, denoting the area of high temperature, is approximately equal to the illuminated area, beyond which the temperature sharply falls. The MLT manifests itself at the center of the illuminated area. Figure $3 \mathrm{~b}$ shows that the heating effect only affected a tiny portion of the fluid and solid domains, because the laser illuminated area had a diameter of several microns. However, the MLT is significant and will adversely affect the accuracy and reliability of the Raman measurement. Besides, the profile of the temperature rise has no obvious distortion as seen in Figure 3a,b, implying negligible contribution from the forced convective heat transfer. These characteristics do not vary with different simulation conditions.
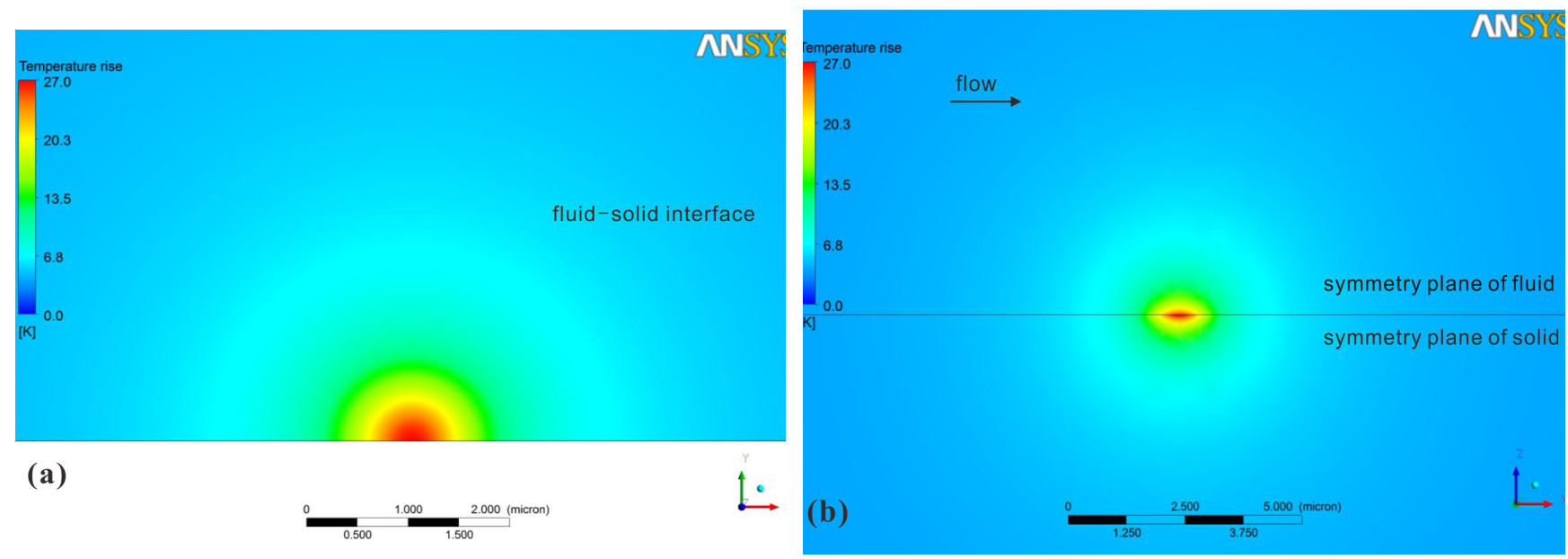

Figure 3. Temperature profile of the computational fluid dynamic (CFD) simulation in such a circumstance: water as the working fluid, $P=10 \mathrm{~mW}, d=1 \mu \mathrm{m}$ and $U=0.01 \mathrm{~m} \cdot \mathrm{s}^{-1}$. (a) At the fluid-solid interface and (b) at the symmetry planes of both fluid and solid domains. 


\subsection{OA Analysis}

The orthogonal array (OA) analysis was conducted in order to extract the sensitive factors to the MLT. Through a series of CFD simulations, the contribution from each level of a design parameter to the MLT was evaluated. The simulations at the same level of each design parameter can be found in Table 1. For example, the second level of parameter B, B2, is present in the 2nd, 7th, 12th, 17th, 22nd, 27th, 32nd, 37th, 42nd and 47th simulations. The mean MLT of the simulations at one level of the design parameter was then calculated and plotted in Figure 4. The MLT is found to be the most sensitive to parameter $\mathrm{A}$, the surface power, for which the difference between the maximum and minimum of the mean MLT is the biggest, followed by the parameter B, the diameter of illuminated area. The other nine parameters all have the data range of no more than $3 \mathrm{~K}$.

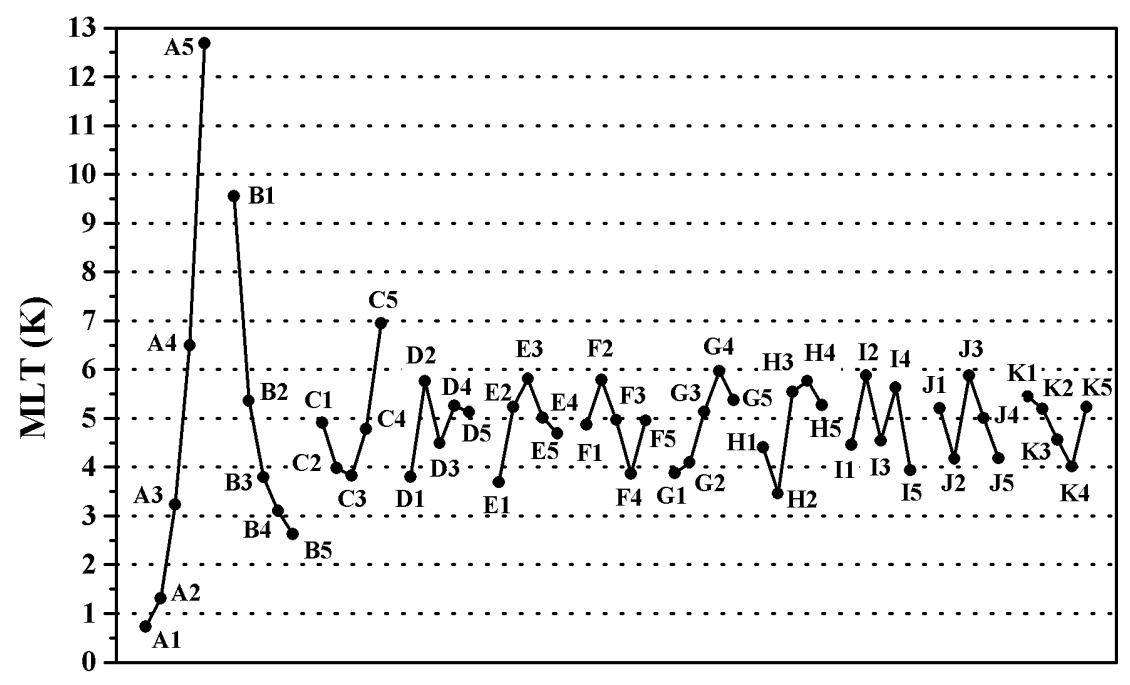

Figure 4. Influence of factors on the maximum local temperature-rise (MLT) for a set of 50 models defined by the orthogonal array (OA) L50.

Analysis of variance (ANOVA) was conducted to further determine the contribution of each factor to the MLT. The sum of squares for each factor is listed in Table 3. The percentage of each SS in the total sum of squares was calculated and presented by way of their contribution. It can be seen that the surface power $P$ contributes $62.6 \%$ to the MLT, and the diameter of illuminated area $d$ contributes $20.5 \%$. Thereby, $P$ and $d$ are considered as the dominant design parameters in the OA. Their relations to the MLT will be further investigated in the following sections. The contribution numbers of $P$ and $d$ are an order magnitude larger than those of the other nine factors. It is believed that both of them directly contribute to the heat flux of Equation (8) at the fluid-solid interface. The transparent fluids were considered to absorb little heat from the visible light. Therefore, the heat source has the strong effect on the laser-induced heating mainly through thermal conduction in the solid. Thus, the temperature in the solid increases significantly, leading to the great difference of temperature in the fluid and solid. The temperature difference drives the convective flow of the fluid. Then, the fluid acquires the heat by forced convective heat transfer. In the factors considering the forced convective heat transfer, fluid mean velocity $U$ makes a slightly greater contribution than do fluid physical properties and microchannel sizes (parameter D-I). 
Table 3. Analysis of variance for the maximum local temperature-rise (MLT).

\begin{tabular}{cccccccccccc}
\hline Factors & $\mathbf{A}$ & $\mathbf{B}$ & $\mathbf{C}$ & $\mathbf{D}$ & $\mathbf{E}$ & $\mathbf{F}$ & $\mathbf{G}$ & $\mathbf{H}$ & $\mathbf{I}$ & $\mathbf{J}$ & $\mathbf{K}$ \\
\hline $\mathrm{SS}^{*}$ & 961.935 & 315.083 & 62.373 & 23.04 & 24.621 & 18.631 & 31.063 & 36.248 & 27.455 & 21.068 & 13.979 \\
Contribution & $62.6 \%$ & $20.5 \%$ & $4.1 \%$ & $1.5 \%$ & $1.6 \%$ & $1.2 \%$ & $2.0 \%$ & $2.4 \%$ & $1.8 \%$ & $1.4 \%$ & $0.9 \%$ \\
\hline \multicolumn{1}{c}{ Note: *: Sum of squares for each factor. }
\end{tabular}

\subsection{Effects of Surface Power and Diameter of Illuminated Area}

The surface power $p$ and diameter of illuminated area $d$ play the dominant roles in the case of the laser-induced heating as discussed above. In order to further investigate their sole contribution, two sets of CFD simulations were carried out after OA analysis with water as the working fluid. Viscosity, density, specific heat and thermal conductivity of water were $0.89 \mathrm{mPa} \cdot \mathrm{s}, 997 \mathrm{~kg} \cdot \mathrm{m}^{-3}, 4.19 \mathrm{~kJ} \cdot \mathrm{kg}^{-1} \cdot \mathrm{K}^{-1}$ and $0.6069 \mathrm{~W} \cdot \mathrm{m}^{-1} \cdot \mathrm{K}^{-1}$, respectively.

In the present simulations, the effect of $p$ was studied in a more systematic way, with the diameter of illuminated area of 1,3 and $5 \mu \mathrm{m}$, and the mean fluid velocity of 0.01 and $0.1 \mathrm{~m} \cdot \mathrm{s}^{-1}$. As shown in Figure 5, the surface power saliently influences the local temperature rise. A surface power of $10 \mathrm{~mW}$ can lead to an MLT up to $26.8 \mathrm{~K}$ with $1 \mu \mathrm{m}$ diameter of illuminated area. Even though the surface power decreases to $0.5 \mathrm{~mW}$, the temperature rise is still appreciable. Moreover, the MLT is noted to be approximately directly proportional to the surface power under all simulation conditions. Their relationship is shown in Equation (10). This discovery can facilitate the correlation of the MLT in the later section. Besides, the MLT cannot be effectively reduced even if the fluid velocity is increased tenfold, indicating a negligibly small effect of the fluid velocity.

$$
\Delta T_{\max } \propto P
$$

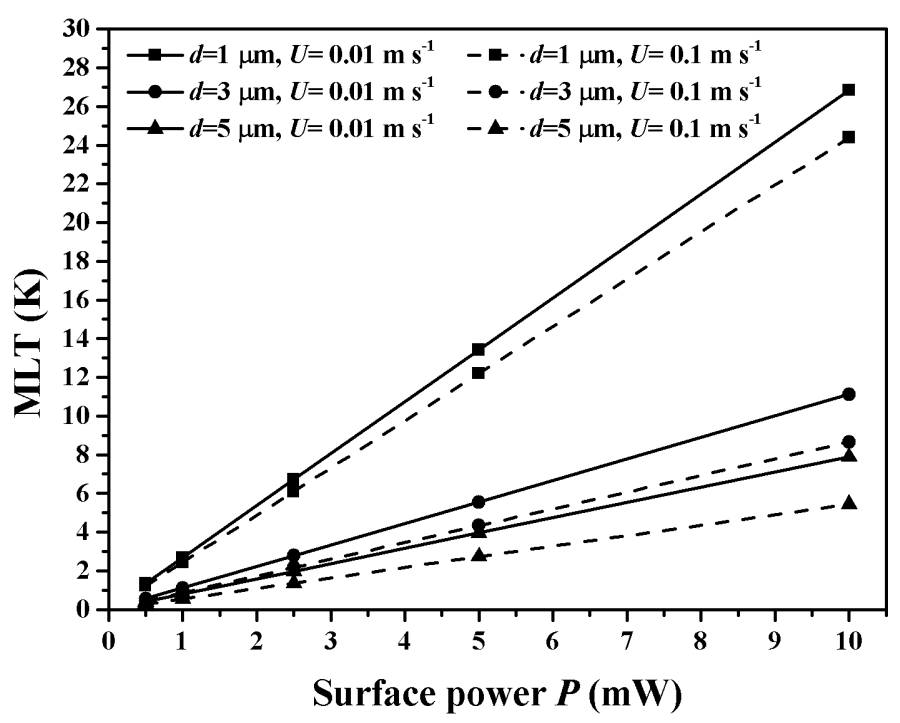

Figure 5. Effect of the surface power at the mean velocity of $0.01 \mathrm{~m} \cdot \mathrm{s}^{-1}$ (solid line) and $0.1 \mathrm{~m} \cdot \mathrm{s}^{-1}$ (dashed line) when the diameter of illuminated areas are $1 \mu \mathrm{m}(\boldsymbol{\bullet}), 3 \mu \mathrm{m}(\bullet)$ and $5 \mu \mathrm{m}(\boldsymbol{\Delta})$, respectively. The microchannel is $800 \mu \mathrm{m}$ wide, $30 \mu \mathrm{m}$ high and $10 \mathrm{~mm}$ long. The chip is $10 \mathrm{~mm}$ wide, $0.4 \mathrm{~mm}$ thick and $10 \mathrm{~mm}$ long. 
The second set of simulation is to present the effect of the diameter of illuminated area $d$ with the surface powers of 10,5 and $0.5 \mathrm{~mW}$, and the mean fluid velocity of 0.01 and $0.1 \mathrm{~m} \cdot \mathrm{s}^{-1}$. The data are plotted in Figure 6. The increase in the diameter of illuminated area leads to an obvious MLT decrease, due to the reduced heat flux over the illuminated area. It can be seen that, on the log-log scale, the data points shows that certain $P$ and $U$ regularly lie along a straight line, indicating an exponential dependence of the MLT on $d$. Through a rough fit, the exponent of all these lines is about -1 , and hence the relationship between the MLT and $d$ is presented in Equation (11). This relationship was also employed to simplify the correlation of the MLT. It should be also pointed out that it is impractical to reduce the MLT by simply increasing the illuminated area during the actual measurement, because increasing the illuminated area sacrifices the accuracy of the Raman measurement.

$$
\Delta T_{\max } \propto d^{-1}
$$

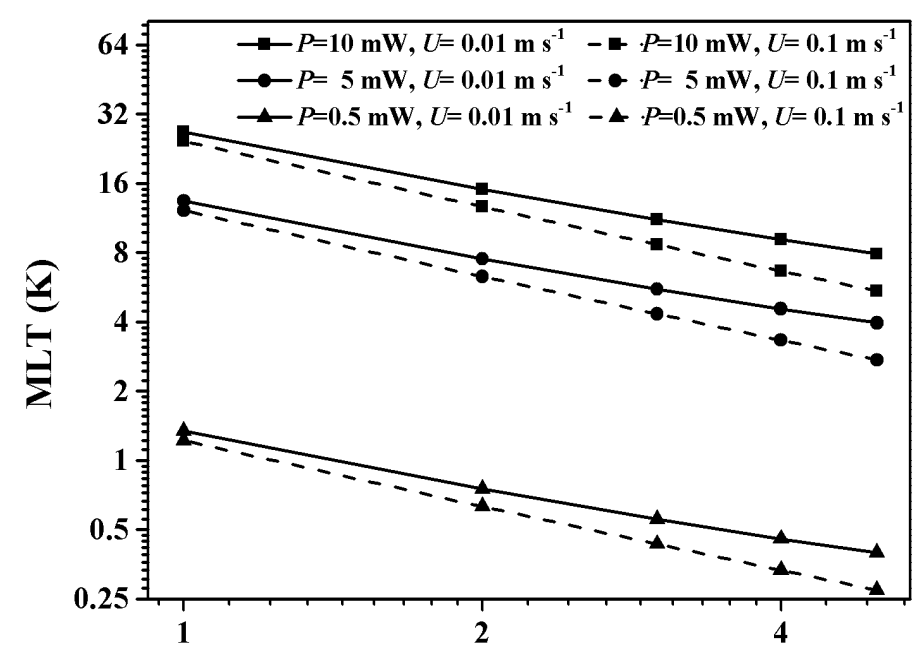

Diameter of illuminated area $d(\mu \mathrm{m})$

Figure 6. Effect of the diameter of illuminated area at the mean velocity of $0.01 \mathrm{~m} \cdot \mathrm{s}^{-1}$ (solid line) and $0.1 \mathrm{~m} \cdot \mathrm{s}^{-1}$ (dashed line) when the surface powers are $10 \mathrm{~mW}(\boldsymbol{\bullet}), 5 \mathrm{~mW}(\bullet)$ and $0.5 \mathrm{~mW}(\boldsymbol{\Delta})$, respectively. The microchannel is $800 \mu \mathrm{m}$ wide, $30 \mu \mathrm{m}$ high and $10 \mathrm{~mm}$ long. The chip is $10 \mathrm{~mm}$ wide, $0.4 \mathrm{~mm}$ thick and $10 \mathrm{~mm}$ long.

\subsection{Effect of Exposure Time}

All the previously discussed simulation results were based on the steady-state assumption, which means they are independent of the exposure time. Therefore, transient simulations were carried out in this section to cover the effect of the exposure time. The aforementioned water data and entire chip geometry (including fluid and solid domain) were used in the simulations. Along with transient results, the steady-state results are also presented to determine the time needed for the MLT to be constant, as shown in Figure 7. It is seen that over $90 \%$ of the MLT increment is established during the first second. The increase in the MLT apparently slows down as the time exceeds $1 \mathrm{~s}$. Note that the minimum exposure time of commercial Raman microscopes is $1 \mathrm{~s}$. Therefore, the cumulative effect of the exposure time is negligible, and the steady-state simulation results can represent the accurate MLT. It is reasonable to use the steady-state CFD simulations to investigate the heating effect. 


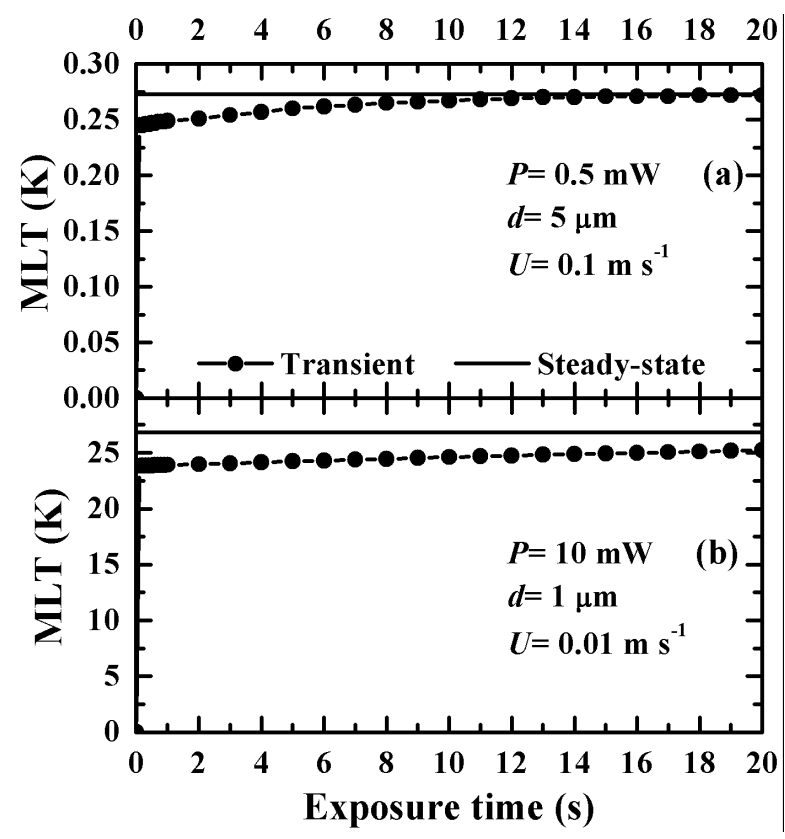

Figure 7. Transient simulation results: MLT v.s. the exposure time under two conditions: (a) $P=0.5 \mathrm{~mW}, d=5 \mu \mathrm{m}, U=0.1 \mathrm{~m} \cdot \mathrm{s}^{-1}$ and (b) $P=10 \mathrm{~mW}, d=1 \mu \mathrm{m}, U=0.01 \mathrm{~m} \cdot \mathrm{s}^{-1}$. The microchannel is $800 \mu \mathrm{m}$ wide, $30 \mu \mathrm{m}$ high and $10 \mathrm{~mm}$ long. The chip is $10 \mathrm{~mm}$ wide, $0.4 \mathrm{~mm}$ thick and $10 \mathrm{~mm}$ long.

\subsection{Experimental Evidence of Temperature Rise}

The diffusion measurements of the toluene/cyclohexane system were conducted to verify the simulation results. The molar fractions of toluene were obtained by the Raman measurement, and were then fit to correlate the diffusion coefficient $D$, as elaborated in our previous work [18]. The microchannel geometry in the corresponding CFD simulations is identical to that in the Raman experimental measurements (see Section 3), and the maximum chip size available with our computer is $25 \mathrm{~mm} \times 25 \mathrm{~mm}$. Since toluene and cyclohexane have similar thermophysical properties, the simulations were carried out only with toluene rather than with both liquids, to reflect the heating process in the actual measurements. The surface power was selected as the experimental variable to demonstrate the effect of a dominant factor. On the contrary, the fluid velocity was also selected to represent the factors with negligible effect on the MLT. Other design parameters were not varied in the experiment due to their negligibly small effect.

Figure 8 illustrates that, with an increase in the surface power, the $D$ value increases considerably, indicating a concomitant temperature rise. This temperature rise depends heavily on the surface power. Similarly, an obvious MLT increase can be found in the simulation results (see Figure 8). Both simulation and experimental results serve as strong evidence that the laser-induced heating effect relies on the surface power significantly. As shown in Figure 9, the $D$ values show negligibly small dependence on mean velocity, meaning that the mean velocity does not play an important role in the temperature rise. Based on simulation results, the mean velocity contributes little to the MLT (see Figure 9). The CFD simulation results are therefore validated by their congruencies with the experimental trends, as shown in Figures 8 and 9. Nevertheless, it should be pointed out that, in this research, the temperature gradient between the liquid in the laser focus and the bulk liquid may result in an undesired thermal diffusion, which may further increase the measured $D$ value. In contrast, the 
temperature-dependent $D$ values reported in the literature were obtained with an exclusive presence of the molecular diffusion. Therefore, it is unreasonable to quantitatively predict the temperature rise by simply comparing $D$ from the present diffusion experiment with that reported in the literature.

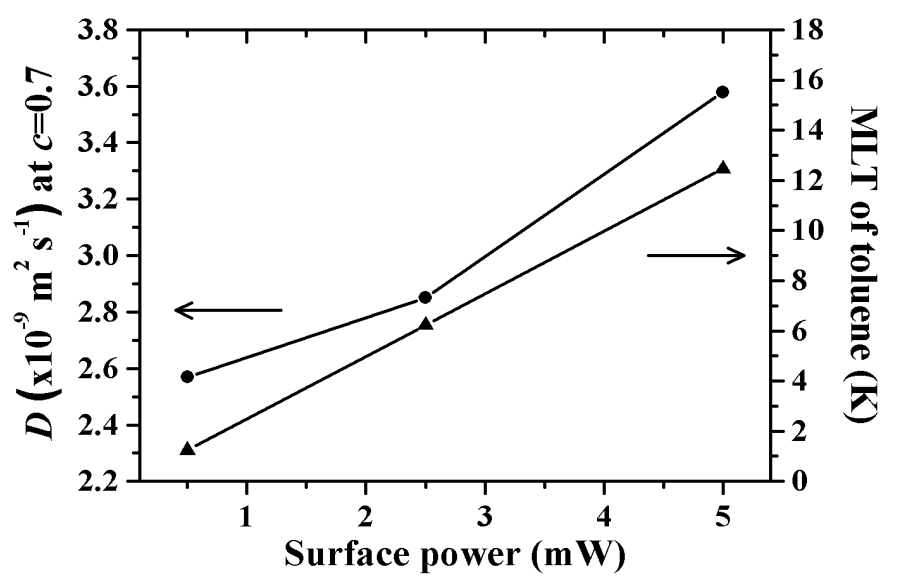

Figure 8. Comparison of simulation and experimental results, in terms of the influence of the surface power on the heating effect. $d=1 \mu \mathrm{m}$ and $U=0.013 \mathrm{~m} \cdot \mathrm{s}^{-1}$.

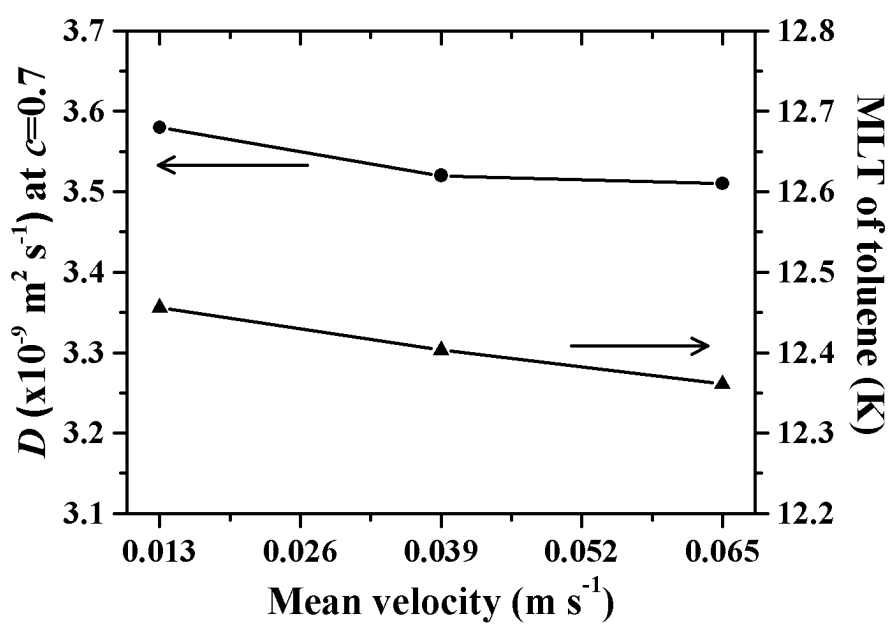

Figure 9. Comparison of simulation and experimental results, in terms of the influence of the mean velocity on the heating effect. $P=5 \mathrm{~mW}$ and $d=1 \mu \mathrm{m}$.

\subsection{Estimation of the Laser-Induced Heating Effect}

Due to the difficulty of fluid temperature measurement in the microchannel, it is important to establish an estimation method for the MLT to determine whether the laser-induced heating effect can be neglected. As discussed in OA analysis, several factors, including the Raman laser parameters, fluid and solid properties, and sizes of the fluid and solid domains, affect the laser-induced heating to various extents, adding to the complexity in establishing the correlations. Factors with weak influences should be neglected for simplification. As mentioned previously in Section 4.1, the convective heat transfer has a minor contribution to the overall conjugate heat transfer. The Maranzana number $M$ was thus introduced in order to evaluate whether parameters in relation to the convective heat transfer can be neglected. $M$ is the ratio of the typical heat flux of axial conduction in the solid walls to the convective heat flux in the fluid: 


$$
M=\frac{\lambda_{\mathrm{s}}}{\lambda_{\mathrm{f}}} \cdot \frac{A_{\mathrm{s}}}{A_{\mathrm{f}}} \cdot \frac{1}{\operatorname{RePr}}
$$

Maranzana et al. [35] proposed that if the axial conduction heat flux is two orders of magnitude smaller than the convective heat flux (i.e., $M<0.01$ ), the axial thermal conduction can be neglected. Therefore, in our case, it can be concluded that when the axial conduction heat flux is over two orders of magnitude higher than the convective heat flux $(M>100)$, the convective heat transfer will be negligible. The thermal conduction dominated conjugate heat transfer means that the fluid temperature develops quickly along the flow direction and very close to the wall temperature. It can be seen in Figure $3 \mathrm{~b}$ that the almost symmetric temperature profiles on the fluid and solid sides. This is a typical feature of thermal conduction dominated. Within the parameter range in the current simulations, $M$ varies between 123 and 588,000. It is reasonable to believe that the thermal conduction in the solid dominates the conjugate heat transfer and the convective heat transfer is negligible. It was found in the $M$ calculation that the variation of the convective heat flux is obviously larger than that of the thermal conduction. The smaller $M$ values all results from the larger convective heat flux. In Equation (12), the denominator as the representative of the convective term was simplified as $2 c_{p \mathrm{f}} \rho_{\mathrm{f}} U A_{\mathrm{f}}^{2} /(h+w)$. It shows that the convective term is proportional to the mean velocity and two fluid physical properties, namely the specific heat and density. Moreover, the physical properties are determined by fluids. For one fluid, it is impossible that the values of the specific heat and density are both at high levels. However, the increased mean velocity indeed leads to a slight enhancement of the convection. It is noted that this term has the relationship with the three power of the channel size. Only increasing the channel size can significantly strengthen the convection, but the size should be limited to the microscale. To conclude, the rule of dominant thermal conduction needs to be confirmed when encountering a large mean velocity or channel size. By considering the great $M$ values in our case, the parameters of the solid rather than the fluid were taken into account in correlating the MLT.

Prior to establishing the correlation, dimensional analysis was developed with the smallest group of necessary parameters. Table 4 lists the three dimensionless groups that were derived using the Buckingham $\pi$ theorem, from the following six parameters: the absorbed power by the solid $P \cdot \alpha$, the diameter of illuminated area $d$, the mean velocity $U$, the thermal conductivity of the solid $\lambda_{s}$, the area of the whole microfluidic chip $L \cdot W$, and the maximum local temperature-rise $\Delta T_{\max }$. Based on nonlinear regression analysis, the dependent variable $\pi_{3}$ can be expressed as follows with $R^{2}=0.980$ :

$$
\pi_{3}=266.764+0.550 \times \pi_{1}+0.000285 \times \pi_{2}
$$

Equation (13) can be converted to the function of $\Delta T_{\max }$ :

$$
\Delta T_{\max }=P \cdot \alpha\left(266.764 \frac{1}{\lambda_{\mathrm{s}} \cdot(L \cdot W)^{0.5}}+0.550 \frac{1}{d \cdot \lambda_{\mathrm{s}}}+0.000285 \frac{1}{U \cdot(L \cdot W)}\right)
$$

This correlation can estimate the MLT within the following parameter ranges: $P$ : $0.5-10 \mathrm{~mW}$, $d: 1-5 \mu \mathrm{m}, U: 0.01-0.1 \mathrm{~m} \cdot \mathrm{s}^{-1}$, and $L \cdot W: 25-225 \mathrm{~mm}^{2}$. Besides, this correlation is applicable to almost all common liquids, such as toluene, ethanol and water, whose physical properties have the ranges of $\rho_{\mathrm{f}}: 494-1584 \mathrm{~kg} \cdot \mathrm{m}^{-3}, \mu_{\mathrm{f}}: 0.1-1.92 \mathrm{mPa} \cdot \mathrm{s}, c_{p \mathrm{f}}: 1.05-4.19 \cdot \mathrm{kJ} \cdot \mathrm{kg}^{-1} \cdot \mathrm{K}^{-1}$ and $\lambda_{\mathrm{f}}: 0.104-0.6069 \mathrm{~W} \cdot \mathrm{m}^{-1} \cdot \mathrm{K}^{-1}$. Above ranges are commonly encountered in the Raman detection of chemical and biological processes. Thereby, the proposed correlation can be widely applied in these fields. 
Table 4. Dimensionless groups for describing the laser-induced heating effect (Unit: SI).

\begin{tabular}{cc}
\hline Dimensionless Group & Expression \\
\hline$\pi_{1}$ & $\left.(L \cdot W)^{1 / 2}\right) / d$ \\
$\pi_{2}$ & $\lambda_{s} /\left(U \cdot(L \cdot W)^{1 / 2}\right)$ \\
$\pi_{3}$ & $\Delta T_{\max } \cdot \lambda_{s} \cdot(L \cdot W)^{1 / 2} /(P \cdot \alpha)$ \\
\hline
\end{tabular}

In addition, in order to ensure the accurate estimation of the MLT using the correlation, it is important to accurately determine the Raman laser parameters, including the surface power $P$ and the diameter of illuminated area $d$, due to their major contributions to the MLT. $P$ can be readily measured by a laser power meter. $d$ can be estimated from the microscopic image of the laser spot when the laser is directly focused on the channel bottom of the silicon microchannel, as discussed in Section 2.1. According to the calculated MLT, it is convenient to identify the laser-induced heating effect and to understand to what extent it affects the Raman measurement. To our knowledge, this is the first correlation pertinent to the MLT prediction in the Raman measurement in a silicon-based microchannel. More experimental data are therefore needed to improve and modify this correlation in future work. What is more, the application will be extended by considering various substrate materials.

\section{Conclusions}

When Raman microscopy is adopted to detect the chemical and biological processes in a silicon microfluidic chip, laser-induced heating effect should be considered. In this paper, three-dimensional CFD simulations were conducted to investigate the heating effect. Based on OA analysis from CFD results, the surface power $P$ was the most influential factor on the MLT, followed by the diameter of illuminated area $d$. This tendency was also verified by a measurement of temperature-sensitive diffusion coefficient using a silicon-based microfluidic chip. The transient CFD simulation found that over $90 \%$ of the MLT was established within the first second of the exposure time, suggesting the reliability of the results based on the steady-state assumption. To quantitatively evaluate the Raman laser-induced heating effect in microfluidic chip, a correlation was established for the first time by dimensional analysis and nonlinear regression analysis to calculate the MLT. Simple in form and convenient for calculation, this correlation can be efficiently applied to Raman measurement in a silicon microfluidic channel.

\section{Acknowledgements}

This study was financially supported by the China Natural Science Foundation (Contract No. 21176069, 21476073), the Fundamental Research Funds for the Central Universities (WG1213011) and Program for Introduced Talents in Shanghai Institute of Technology (YJ2012-14).

\section{Author Contributions}

Ying Lin was responsible for numerical simulation and experimental validation. Xinhai Yu and Zhenyu Wang analyzed the numerical and experimental results. Both Shan-Tung Tu and Zhengdong Wang developed the research plan. Ying Lin and Xinhai Yu wrote the main manuscript and all authors reviewed the manuscript. 


\section{Nomenclature}

A cross-sectional area along the axial direction, $\mathrm{m}^{2}$

$c_{p} \quad$ specific heat, $\mathrm{kJ} \cdot \mathrm{kg}^{-1} \cdot \mathrm{K}^{-1}$

$d \quad$ diameter of illuminated area, $\mu \mathrm{m}$

$D \quad$ diffusion coefficient, $\mathrm{m}^{2} \cdot \mathrm{s}^{-1}$

$h_{\mathrm{fc}} \quad$ free convective heat transfer coefficient, $\mathrm{W} \cdot \mathrm{m}^{-2} \cdot \mathrm{K}^{-1}$

$h \quad$ height of the microchannel, $\mu \mathrm{m}$

I laser intensity, $\mathrm{W} \cdot \mathrm{m}^{-2}$

$L \quad$ length of the microfluidic chip, $\mathrm{mm}$

$p$ pressure, $\mathrm{Pa}$

$P \quad$ surface power, $\mathrm{mW}$

$q \quad$ heat flux, $\mathrm{W} \cdot \mathrm{m}^{-2}$

$r \quad$ the distance with the spot center, $\mu \mathrm{m}$

$T$ temperature, $\mathrm{K}$

$\Delta T_{\max }$ maximum local temperature-rise of fluid, abbreviated as MLT, $\mathrm{K}$

$u \quad$ velocity vector

$U \quad$ mean velocity, $\mathrm{m} \cdot \mathrm{s}^{-1}$

$w \quad$ width of the microchannel, $\mu \mathrm{m}$

$W \quad$ width of the microfluidic chip, $\mathrm{mm}$

Dimensionless groups

$M \quad$ Maranzana number

$\operatorname{Pr} \quad$ Prandtl number, $c_{p \mathrm{f}} \mu_{\mathrm{f}} \lambda_{\mathrm{f}}$

Re Reynolds number, $10^{-6} \rho_{\mathrm{f}} U 2(h w) /(h+w) / \mu_{\mathrm{f}}$

Greek symbols

$\alpha \quad$ absorption coefficient

$\lambda \quad$ thermal conductivity, $\mathrm{W} \cdot \mathrm{m}^{-1} \cdot \mathrm{K}^{-1}$

$\mu \quad$ dynamic viscosity, $\mathrm{mPa} \mathrm{s}$

$\rho \quad$ density, $\mathrm{kg} \cdot \mathrm{m}^{-3}$

$\omega_{0} \quad$ radius of laser spot, $\mu \mathrm{m}$

\section{Subscripts}

0 the center of laser spot

a ambient

f fluid

s solid

\section{Conflicts of Interest}

The authors declare no conflict of interest.

\section{References}

1. Kamholz, A.E.; Weigl, B.H.; Finlayson, B.A.; Yager, P. Quantitative analysis of molecular interaction in a microfluidic channel: The T-sensor. Anal. Chem. 1999, 71, 5340-5347. 
2. DeMello, A.J. Control and detection of chemical reactions in microfluidic systems. Nature 2006, 442, 394-402.

3. McClain, M.A.; Culbertson, C.T.; Jacobson, S.C.; Allbritton, N.L.; Sims, C.E.; Ramsey, J.M. Microfluidic devices for the high-throughput chemical analysis of cells. Anal. Chem. 2003, 75, 5646-5655.

4. Mazutis, L.; Baret, J.C.; Treacy, P.; Skhiri, Y.; Araghi, A.F.; Ryckelynck, M.; Taly, V.; Griffiths, A.D. Multi-step microfluidic droplet processing: Kinetic analysis of an in vitro translated enzyme. Lab Chip 2009, 9, 2902-2908.

5. Ohno, K.; Tachikawa, K.; Manz, A. Microfluidics: Applications for analytical purposes in chemistry and biochemistry. Electrophoresis 2008, 29, 4443-4453.

6. Fräulin, C.; Rinke, G.; Dittmeyer, R. Characterization of a new system for space-resolved simultaneous in situ measurements of hydrocarbons and dissolved oxygen in microchannels. Microfluid. Nanofluid. 2014, 16, 149-157.

7. Pully, V.V.; Lenferink, A.; van Manen, H.J.; Subramaniam, V.; van Blitterswijk, C.A.; Otto, C. Microbioreactors for raman microscopy of stromal cell differentiation. Anal. Chem. 2010, 82, 1844-1850.

8. Egawa, T.; Durand, J.L.; Hayden, E.Y.; Rousseau, D.L.; Yeh, S.R. Design and evaluation of a passive alcove-based microfluidic mixer. Anal. Chem. 2009, 81, 1622-1627.

9. Sarrazin, F.; Salmon, J.B.; Talaga, D.; Servant, L. Chemical reaction imaging within microfluidic devices using confocal raman spectroscopy: The case of water and deuterium oxide as a model system. Anal. Chem. 2008, 80, 1689-1695.

10. Fletcher, P.D.I.; Haswell, S.J.; Zhang, X.L. Monitoring of chemical reactions within microreactors using an inverted raman microscopic spectrometer. Electrophoresis 2003, 24, 3239-3245.

11. Zhang, W.; Li, B.; Chen, L.; Wang, Y.; Gao, D.; Ma, X.; Wu, A. Brushing, a simple way to fabricate sers active paper substrates. Anal. Methods 2014, 6, 2066-2071.

12. Ndukaife, J.C.; Mishra, A.; Guler, U.; Nnanna, A.G.A.; Wereley, S.T.; Boltasseva, A. Photothermal heating enabled by plasmonic nanostructures for electrokinetic manipulation and sorting of particles. ACS Nano 2014, 8, 9035-9043.

13. Wang, Y.; Yan, B.; Chen, L. Sers tags: Novel optical nanoprobes for bioanalysis. Chem. Rev. 2013, $113,1391-1428$.

14. Li, Q.-L.; Li, B.-W.; Wang, Y.-Q. Surface-enhanced raman scattering microfluidic sensor. RSC Adv. 2013, 3, 13015-13026.

15. Everall, N.J. Modeling and measuring the effect of refraction on the depth resolution of confocal raman microscopy. Appl. Spectrosc. 2000, 54, 773-782.

16. Everall, N.J. Confocal raman microscopy: Why the depth resolution and spatial accuracy can be much worse than you think. Appl. Spectrosc. 2000, 54, 1515-1520.

17. Salmon, J.B.; Ajdari, A.; Tabeling, P.; Servant, L.; Talaga, D.; Joanicot, M. In situ raman imaging of interdiffusion in a microchannel. Appl. Phys. Lett. 2005, 86, 094106.

18. Lin, Y.; Yu, X.; Wang, Z.; Tu, S.-T.; Wang, Z. Measurement of temperature-dependent diffusion coefficients using a confocal raman microscope with microfluidic chips considering laser-induced heating effect. Anal. Chim. Acta 2010, 667, 103-112. 
19. Lo, H.W.; Compaan, A. Raman measurements of temperature during $\mathrm{cw}$ laser heating of silicon. J. Appl. Phys. 1980, 51, 1565.

20. Kouteva-Arguirova, S.; Arguirov, T.; Wolfframm, D.; Reif, J. Influence of local heating on micro-raman spectroscopy of silicon. J. Appl. Phys. 2003, 94, 4946-4949.

21. Wu, X.M.; Yu, J.Y.; Ren, T.L.; Liu, L.T. Micro-raman spectroscopy measurement of stress in silicon. Microelectron. J. 2007, 38, 87-90.

22. Georgi, C.; Hecker, M.; Zschech, E. Effects of laser-induced heating on raman stress measurements of silicon and silicon-germanium structures. J. Appl. Phys. 2007, 101, 123104.

23. Suthar, K.J.; Patten, J.; Dong, L.; Abdel-Aal, H. Estimation of Temperature Distribution in Silicon During Micro Laser Assisted Machining. In Proceedings of the ASME 2008 International Manufacturing Science and Engineering Conference, Evanston, IL, USA, 7-10 October 2008; pp. 301-309.

24. Downes, A.; Salter, D.; Elfick, A. Heating effects in tip-enhanced optical microscopy. Opt. Express 2006, 14, 5216-5222.

25. Hung, M.-S.; Kurosawa, O.; Washizu, M. Single DNA molecule denaturation using laser-induced heating. Mol. Cell. Probes 2012, 26, 107-112.

26. Kim, H.; Vishniakou, S.; Faris, G.W. Petri dish pcr: Laser-heated reactions in nanoliter droplet arrays. Lab Chip 2009, 9, 1230-1235.

27. Zhao, R.; Marshall, M.; Alemán, E.A.; Lamichhane, R.; Feig, A.; Rueda, D. Laser-assisted single-molecule refolding (lasr). Biophys. J. 2010, 99, 1925-1931.

28. Baaske, P.; Duhr, S.; Braun, D. Melting curve analysis in a snapshot. Appl. Phys. Lett. 2007, 91, 133901.

29. Gallardo, A.; Spells, S.; Navarro, R.; Reinecke, H. Confocal raman microscopy: How to correct depth profiles considering diffraction and refraction effects. J. Raman Spectrosc. 2007, 38, 880-884.

30. Everall, N. Depth profiling with confocal raman microscopy, part II. Spectroscopy 2004, 19, 16-26.

31. Baldwin, K.J.; Batchelder, D.N. Confocal raman microspectroscopy through a planar interface. Appl. Spectrosc. 2001, 55, 517-524.

32. Bruneel, J.L.; Lassegues, J.C.; Sourisseau, C. In-depth analyses by confocal raman microspectrometry: Experimental features and modeling of the refraction effects. J. Raman Spectrosc. 2002, 33, 815-828.

33. Tiselj, I.; Hetsroni, G.; Mavko, B.; Mosyak, A.; Pogrebnyak, E.; Segal, Z. Effect of axial conduction on the heat transfer in micro-channels. Int. J. Heat Mass Transf. 2004, 47, 2551-2565.

34. Engineering ToolBox. Available online: http://www.engineeringtoolbox.com. (accessed on 24 June 2015).

35. Maranzana, G.; Perry, I.; Maillet, D. Mini- and micro-channels: Influence of axial conduction in the walls. Int. J. Heat Mass Transf. 2004, 47, 3993-4004.

(C) 2015 by the authors; licensee MDPI, Basel, Switzerland. This article is an open access article distributed under the terms and conditions of the Creative Commons Attribution license (http://creativecommons.org/licenses/by/4.0/) 